\title{
Evaluation of method of preparation of passive diffusion tubes for measurement of ambient nitrogen dioxide
}

\author{
Richard P. Hamilton and Mathew R. Heal* \\ School of Chemistry, University of Edinburgh, West Mains Road, Edinburgh, UK EH9 3JJ. \\ E-mail: m.heal@ed.ac.uk; Fax: +44 (0)1316504743; Tel: +44 (0)1316504764
}

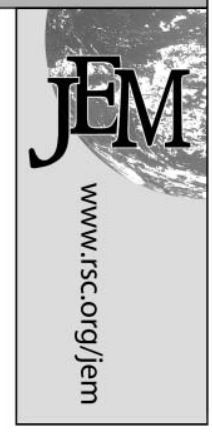

Received 25th September 2003, Accepted 24th October 2003

First published as an Advance Article on the web 17th November 2003

This study was carried out in response to suggestions that the measurement of $\mathrm{NO}_{2}$ by Palmes-type passive diffusion tubes (PDT) is affected by the method of preparation of the triethanolamine (TEA) absorbent coating on the grids. The following combinations of factors were investigated: TEA solvent (acetone or water), volume composition of TEA in solvent ( $50 \%$ or $20 \%$ ), and grid coating method (dipping in solution prior to assembly or pipetting solution on after assembly). Duplicate PDTs prepared by each of the 8 methods were exposed in parallel, in urban air, for a total of 80 separate 1 week exposures. $\mathrm{NO}_{2}$ concentrations derived from PDTs prepared by pipetting methods were significantly less precise than concentrations from dipping methods, with mean RSDs for duplicate measurements of $13.8 \%$ and $8.5 \%$, respectively ( $n=316$ each category). Pipetting methods using solutions of 50\% TEA composition were particularly imprecise (mean RSD 17.2\%). Data from PDTs prepared by pipetting methods were systematically more poorly correlated with each other and with data from co-located chemiluminescence analysers, than corresponding data from PDTs prepared by dipping methods, indicating that more consistent accuracy was also obtained by the latter PDTs. The statistical evidence suggested that PDTs prepared by pipetting 50\% TEA in water generally gave lower $\mathrm{NO}_{2}$ concentrations. Although this is in agreement with a previous study, it is also possible that such an observation here may be a statistical artefact given the demonstrably poorer precision of this method. The general tendency of PDTs to show positive bias in $\mathrm{NO}_{2}$ measurement in urban air in 1 week exposures was again evident in this study (mean biases at roadside and urban centre locations of $+35 \%(n=475)$ and $+18 \%(n=112)$, respectively) consistent with augmentation of within-tube $\mathrm{NO}_{2}$ flux by chemical reaction between co-diffusing $\mathrm{NO}$ and $\mathrm{O}_{3}$. Overall, it is recommended that the pipetting method of PDT grid preparation is avoided, or at least investigated further, because of the apparent degradation in precision and accuracy of $\mathrm{NO}_{2}$ measurement. Potential reasons for the effect are discussed.

\section{Introduction}

Nitrogen dioxide $\left(\mathrm{NO}_{2}\right)$ is regarded as an air pollutant because of its adverse effect on human health and its contribution to the formation of low-level ozone. Consequently, in most countries, permitted concentrations of ambient $\mathrm{NO}_{2}$ are subject to legislation. The UK, following the EU and the WHO, has an annual average limit for $\mathrm{NO}_{2}$ of $40 \mu \mathrm{g} \mathrm{m}^{-3}$, equivalent to $21 \mathrm{ppb}$ (parts per billion by volume) at ambient air pressure and temperature. Routine monitoring of $\mathrm{NO}_{2}$ is therefore required to assess compliance with air quality standards. Such monitoring is particularly important for $\mathrm{NO}_{2}$ because the majority of $\mathrm{NO}_{2}$ in ambient air is formed from chemical oxidation of primary emissions of nitric oxide, NO. The rate of this oxidation is influenced by many factors ranging from localscale meteorological dispersion and mixing, to regional scale transport of ozone $\left(\mathrm{O}_{3}\right)$ oxidant, making it difficult to predict $\mathrm{NO}_{2}$ spatial fields.

Continuous analysers, such as the chemiluminescence analyser, are usually specified as the reference method for measurement of $\mathrm{NO}_{2}$, but passive diffusion samplers (particularly the Palmes-type passive diffusion tube, ${ }^{1}$ PDT) remain widely used for indicative assessment of spatial variations of longer-term average $\mathrm{NO}_{2}$ concentrations because of their ease of use and low cost. For example, an $\mathrm{NO}_{2}$ concentration map for the entire UK is interpolated from a national network of PDTs. $^{2}$

The fundamental principle of PDT measurement is that $\mathrm{NO}_{2}$ molecules are captured by triethanolamine (TEA) absorbent, $\mathrm{N}\left(\mathrm{CH}_{2} \mathrm{CH}{ }_{2} \mathrm{OH}\right)_{3}$, coating the grids inside the closed end of the tube, with the rate of capture of $\mathrm{NO}_{2}$ determined by the diffusive flux along the tube. Since TEA is extremely viscous at room temperature, it is dissolved in a solvent for application to the PDT grids. After exposure, trapped $\mathrm{NO}_{2}$ is extracted into aqueous solution as nitrite ions, $\mathrm{NO}_{2}^{-}$, and quantified.

While it is accepted that $\mathrm{NO}_{2}$ PDTs are not as precise as continuous analysers, considerable debate remains regarding the accuracy of $\mathrm{NO}_{2}$ PDTs in the field. This debate surrounds the significance or not of specific factors giving rise to specific biases in deriving $\mathrm{NO}_{2}$ concentrations. Some investigators have concluded that $\mathrm{NO}_{2}$ PDTs are accurate within acceptable tolerances of precision, ${ }^{3}$ whilst others have argued that specific phenomena such as wind-induced turbulence at the entrance to the tube, ${ }^{4}$ or chemical production of additional $\mathrm{NO}_{2}$ by codiffusing $\mathrm{NO}$ and $\mathrm{O}_{3}$ within the tube ${ }^{5,6}$ contribute to an effective over-measurement of $\mathrm{NO}_{2}$ by PDTs, or that an exposure-duration related loss of trapped $\mathrm{NO}_{2}$ contributes to an effective under-measurement. ${ }^{7}$

More recently, two studies ${ }^{8,9}$ have suggested that the accuracy of $\mathrm{NO}_{2}$ PDT measurement may also be affected by the way in which the TEA absorbent at the end of the tube is prepared. The observation has potentially major consequences for comparing $\mathrm{NO}_{2}$ PDT data since there is no generallyrecognised standard method of tube preparation. The variables in preparation are that the TEA absorbent may be dissolved in acetone or deionised water, in volume ratios ranging from $20 \%-50 \%$, and the solution applied to the grids either by dipping the grids into the solution prior to PDT assembly, or by pipetting a known volume of solution onto the grids after assembly. The report ${ }^{9}$ produced by NETCEN, the UK body currently responsible for collating and validating data from the UK national network of $\mathrm{NO}_{2}$ PDTs, recommended that the 
preferred preparation method was to pipette an unspecified volume of a $20 \%$ TEA:water solution onto grids already assembled within the PDT cap. The recommendation was based on the observation that this preparation method yielded $\mathrm{NO}_{2}$ measurements closest in value to those of a chemiluminescence analyser, whilst PDTs prepared by dipping grids into a 50\% TEA:acetone solution (probably the most widespread method), or by pipetting a $50 \%$ TEA:water solution onto assembled grids, were reported to yield $\mathrm{NO}_{2}$ measurements consistently higher, or lower, respectively, than the analyser. The study was conducted in a laboratory chamber. The field investigation by Kirby et al. ${ }^{8}$ also reported that tubes prepared by pipetting $50 \%$ TEA:water solution onto assembled grids yielded lower $\mathrm{NO}_{2}$ measurement but that there was no difference in $\mathrm{NO}_{2}$ measurement from tubes prepared using smaller volume ratios of TEA in water or using grids dipped in $50 \%$ TEA in acetone. Conversely, a recent review of routine PDT data from UK local authority networks reported no discernible effect of tube preparation method on PDT measurements. ${ }^{10}$

One difficulty in trying to rationalise observations concerning the effect, or not, of absorbent preparation method is uncertainty regarding the actual mechanism of $\mathrm{NO}_{2}$ complexation by TEA. A mechanism proposed by Glasius et al.,${ }^{11}$ that yields a 1:1 ratio between $\mathrm{NO}_{2}$ in air and $\mathrm{NO}_{2}{ }^{-}$in solution, is shown in eqn. (1):

$$
\begin{aligned}
& 2 \mathrm{NO}_{2}+\mathrm{N}\left(\mathrm{CH}_{2} \mathrm{CH}_{2} \mathrm{OH}\right)_{3}+2 \mathrm{OH}^{-} \rightarrow 2 \mathrm{NO}_{2}{ }^{-}+ \\
& { }^{-} \mathrm{O}-{ }^{+} \mathrm{N}\left(\mathrm{CH}_{2} \mathrm{CH}_{2} \mathrm{OH}\right)_{3}+\mathrm{H}_{2} \mathrm{O}
\end{aligned}
$$

The required hydroxyl ions are postulated to arise from the dissociation of TEA in water molecules present in the air, so the reaction will not take place in completely dry air, in accord with observations that TEA hydration is important for quantitative $\mathrm{NO}_{2}$ sampling. ${ }^{12}$ The $\mathrm{OH}^{-}$ions may also derive from water molecules in the absorbent solution itself (if present), and it has been suggested that the extent of this process may account for the apparent lower trapping efficiency of different molar ratio TEA:water absorbent solutions. ${ }^{8}$

It is not clear from the studies cited above whether the physical process itself of coating grids (i.e. dipping or pipetting) contributes to differences in $\mathrm{NO}_{2}$ measurement. It is also difficult to rationalise how different preparation methodologies may influence measurement. Given the ambiguity of the above studies and the continued importance of PDTs for ambient $\mathrm{NO}_{2}$ measurement, the impetus for the current work was a detailed investigation of whether, and how, absorbent preparation method affects $\mathrm{NO}_{2}$ concentrations derived from PDTs. Eight different grid preparation methods were compared in simultaneous exposures in urban air. Uniquely, this study also compared a sub-set of the PDT $\mathrm{NO}_{2}$ measurements with the $\mathrm{NO}_{2}$ concentration predicted to be measured by a PDT when within-tube production of $\mathrm{NO}_{2}$ along the diffusion path of the tube is also taken into account. ${ }^{5}$ To limit the impact on data interpretation of the further confounding issue of the loss of nitrite evident during longer exposure periods, ${ }^{7}$ all exposures were of 1 week duration only.

\section{Experimental section}

Standard Palmes-type acrylic diffusion tubes from Gradko International were used (length $7.1 \mathrm{~cm}$, internal cross-sectional area $0.92 \mathrm{~cm}^{2}$, two stainless steel grids per tube). Tubes, caps and grids were cleaned thoroughly before use.

Eight different methods of applying TEA to PDT grids were compared, comprising all combinations of two choices of three two-level factors: TEA solvent (acetone or deionised water), TEA concentration $(50 \%$ or $20 \%$ by volume in the solvent), and application method of solution to grid (dipping or pipetting).
Table 1 Identifiers for the different combinations of absorbent solution composition and grid preparation methods used in this work

\begin{tabular}{lll}
\hline & \multicolumn{2}{l}{ Grid preparation method } \\
\cline { 2 - 3 } Absorbent solution composition & Dipped & Pipetted \\
\hline $50 \%$ TEA, $50 \%$ acetone & $\mathrm{A}$ & $\mathrm{E}$ \\
$20 \%$ TEA, 80\% acetone & $\mathrm{B}$ & $\mathrm{F}$ \\
$50 \%$ TEA, 50\% deionised water & $\mathrm{C}$ & $\mathrm{G}$ \\
$20 \%$ TEA, 80\% deionised water & $\mathrm{D}$ & $\mathrm{H}$ \\
\hline
\end{tabular}

The preparation methods, and their labelling nomenclature, are summarised in Table 1. For dipped method preparations, grids were immersed in the appropriate solution for approximately $5 \mathrm{~min}$, laid on tissue paper, and patted gently with another piece of tissue paper to remove excess solution. Tubes were then assembled and capped. For pipetted methods, a volume of $50 \mu \mathrm{l}$ of solution was pipetted directly onto the two grids within the capped end of a tube already assembled. Tubes were left to stand for approximately $3 \mathrm{~min}$ before the remaining open end was capped.

Tubes were exposed for 1 week at three sites in central Edinburgh: Princes Street gardens (PSt), Castle Street (CSt), and Haymarket (HMt). The PSt site is classified as an urban centre (city centre but not roadside), while CSt and Hmt are roadside sites (1-5 $\mathrm{m}$ from a busy road).

PDTs were always deployed in duplicate. The eight pairs of tubes (plus a field blank of method A) were arranged in random order at each site, adjacent to the inlet of a $\mathrm{NO}_{x}$ chemiluminescence analyser. The PSt site also had an $\mathrm{O}_{3}$ analyser. Hourly averaged $\mathrm{NO}, \mathrm{NO}_{2}$ and $\mathrm{O}_{3}$ data at this site were used as input to a numerical model ${ }^{5,13}$ to calculate the amount of additional $\mathrm{NO}_{2}$ created and trapped within the PDTs, during each exposure, by chemical reaction between $\mathrm{NO}$ and $\mathrm{O}_{3}$ also diffusing inside the tube.

PDT exposures were carried out between November 2001 and March 2002 and again between November 2002 and March 2003, yielding data for a total of 301 week exposures for each preparation method at each site, except for the PSt site at which the continuous analyser ceased operation in December 2002.

After exposure, trapped nitrite in the tubes was extracted into deionised water and quantified by the standard sulfanilamide/NEDA colorimetric method (absorbance measurement at $540 \mathrm{~nm}$ ). Independent duplicate sets of nitrite calibration standards were prepared each week and the average ambient $\left[\mathrm{NO}_{2}\right]$ during the exposure calculated from the $\mathrm{NO}_{2}{ }^{-}$ calibration curves using $0.154 \mathrm{~cm}^{2} \mathrm{~s}^{-1}$ as the diffusion coefficient of $\mathrm{NO}_{2}$ in air.

\section{Results}

\subsection{Precision}

The precision of the PDT measurements was assessed before addressing issues of PDT accuracy. Scatter plots of all the duplicate $\mathrm{NO}_{2}$ measurements are shown in Figs. 1a and b, segregated into dipped and pipetted methods of preparation, respectively.

Fig.1 clearly shows that measurement precision from tubes prepared by dipping methods A-D is considerably better than precision from tubes prepared by pipetting methods E-H. Precision is particularly poor for methods E and G (Fig. 1b) in which an absorbent solution of $50 \%$ TEA in acetone or water, respectively, is pipetted directly onto the grids after PDT assembly. The two other pipetting preparation methods $\mathrm{F}$ and $\mathrm{H}$ show better precision although instances of poor precision remain.

Table 2 summarises the precision RSD data by preparation 

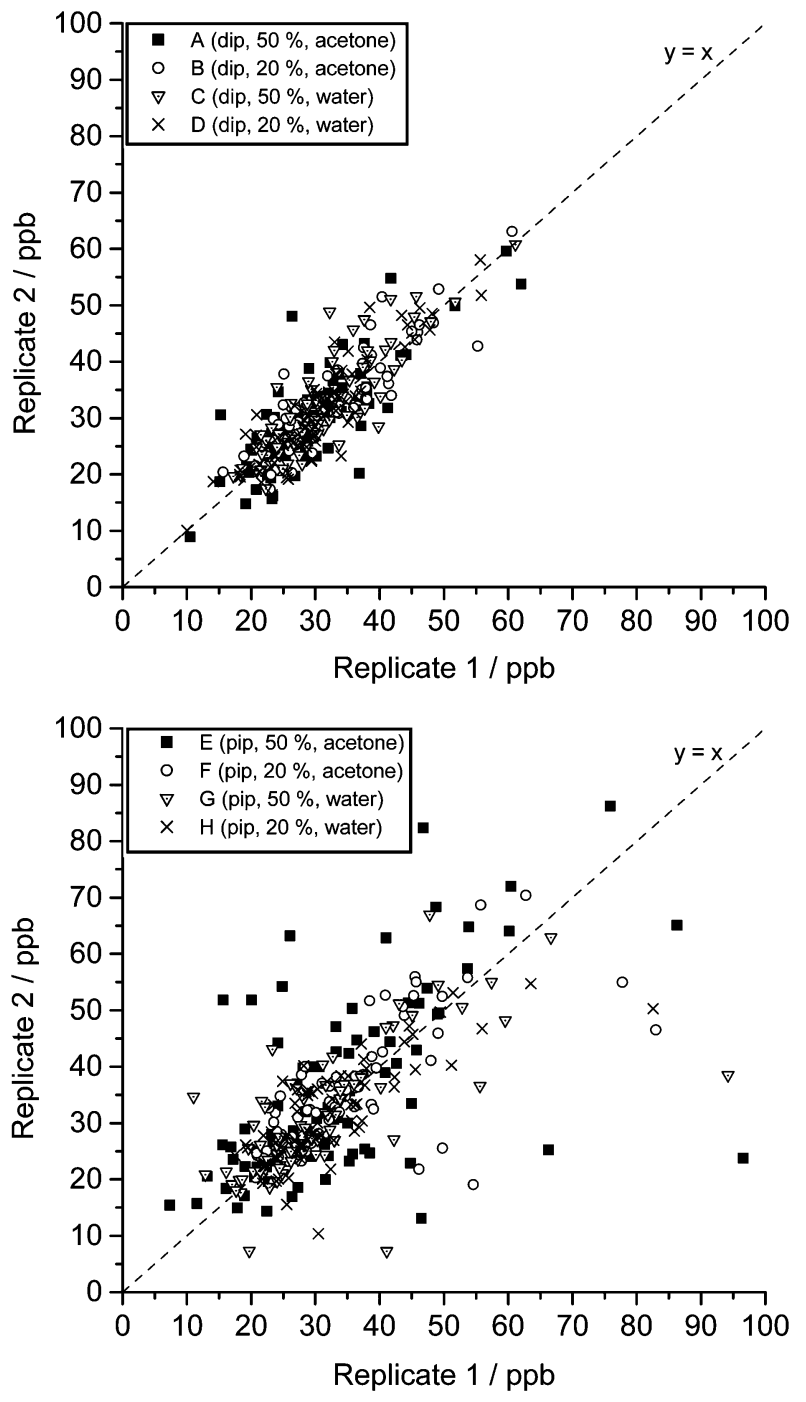

Fig. 1 Scatter plot of duplicate determinations of $\mathrm{NO}_{2}$ concentration for PDTs prepared by (a) all dipping methods, (b) all pipetting methods. Tubes in each replicate pair are randomly assigned the label 1 or 2 .

method. Method E has the worst precision, with mean RSD of $20.9 \%$. The mean RSD of method $\mathrm{G}$ is also high $(13.5 \%)$, although a median RSD of $8.2 \%$ indicates that the mean is adversely affected by some instances of very poor precision (visible in Fig. 1b). The three most precise methods are dipping methods. The mean and median RSD values for all dipping methods $(8.5 \%$ and $6.7 \%$, respectively) are significantly better than the corresponding values $(13.8 \%$ and $9.0 \%)$ for all pipetting methods. With the exception of methods $\mathrm{E}$ and $\mathrm{G}$,

Table 2 Summary of duplicate precision data for $\mathrm{NO}_{2}$ measurement using different tube preparation methods

\begin{tabular}{lrcr}
\hline Preparation method & Mean \% RSD & Median \% RSD & $n$ \\
\hline A (dip, 50\%, acetone) & 10.4 & 7.7 & 80 \\
B (dip, 20\%, acetone) & 7.7 & 6.5 & 80 \\
C (dip, 50\%, water) & 8.2 & 7.4 & 77 \\
D (dip, 20\%, water) & 7.7 & 5.3 & 79 \\
E (pip, 50\%, acetone) & 20.9 & 14.1 & 79 \\
F (pip, 20\%, acetone) & 10.4 & 7.4 & 80 \\
G (pip, 50\%, water) & 13.5 & 8.2 & 87 \\
H (pip, 20\%, water) & 10.6 & 7.5 & 316 \\
All dipped & 8.5 & 6.7 & 316 \\
All pipetted & 13.8 & 9.0 & 632 \\
All & 11.2 & 7.8 & \\
\hline
\end{tabular}

mean RSD values of 7.7-10.6\% for each of the other 6 methods compare well with previously quoted mean RSD values of $8 \%,{ }^{14} \sim 10 \%{ }^{15}$ and $<4 \%{ }^{6}$ for PDT precision.

\subsection{Accuracy}

In order to avoid any subjectivity, the mean of each duplicate pair was used as the measure of $\mathrm{NO}_{2}$ concentration for all PDT data presented in the rest of this paper. No data exclusion criteria were applied.

Time-series comparisons between $\mathrm{NO}_{2}$ concentrations derived from each PDT preparation method and the colocated chemiluminescence analyser for all exposure periods are shown in Fig. 2. The entire dataset comprises up to 80 exposures for each preparation method.

The first observation from Fig. 2 is the highly significant trend for PDT $\mathrm{NO}_{2}$ values to exceed the exposure-average chemiluminescence $\mathrm{NO}_{2}$ concentration (positive bias), regardless of the PDT preparation method ( $P<0.001$, paired $t$-tests). The mean overestimation of analyser $\mathrm{NO}_{2}$ by PDT across all data $(n=587)$ is $32 \%$. The mean overestimations by PDT at each site are $18 \%$ for PSt $(n=112), 41 \%$ for CSt $(n=238)$ and $29 \%$ for HMt $(n=237)$.

Fig. 2a also shows the model-derived $\mathrm{NO}_{2}$ concentration expected for a PDT at the PSt site when additional within-tube chemical production of $\mathrm{NO}_{2}$ (from reaction between the known ambient concentrations of $\mathrm{NO}$ and $\mathrm{O}_{3}$ during each exposure period) is also included. The average ratio between the modelled PDT $\mathrm{NO}_{2}$ concentration and analyser $\mathrm{NO}_{2}$ concentration for this sub-set of exposures is $1.26(n=14$, range 1.131.40 ), confirming again the intrinsic capacity for PDTs to significantly overestimate $\mathrm{NO}_{2}$ because of within-tube chemistry at locations near fluctuating strong sources of $\mathrm{NO}$ (e.g. near roads).$^{5-7}$ The average PDT:analyser $\mathrm{NO}_{2}$ ratio, regardless of PDT preparation method, for exposures for which a modelled value is available is $1.18(n=112)$, which compares well with the mean ratio of 1.26 predicted by the chemical overestimation model. (Note that the observed ratio is likely to be slightly lower, on average, than the model-predicted ratio because of some exposure-duration decline in PDT efficacy even over the 1 week exposures used in this study ${ }^{7}$ ). In fact, for this sub-set of PDT data which can be compared with modelpredicted values, $\mathrm{NO}_{2}$ concentrations derived from PDT methods $\mathrm{A}-\mathrm{F}$ do not differ significantly from model concentrations, whilst concentrations from methods $\mathrm{G}$ and $\mathrm{H}$ are significantly lower (paired $t$-test).

The smaller positive bias, on average, of PDTs at the PSt site, compared with the CSt and HMt sites is again expected, since the former site is situated further from the roadside source of $\mathrm{NO}$ allowing more time for oxidation of $\mathrm{NO}$ to $\mathrm{NO}_{2}$ in the air mass before it enters the tube.

The Spearman rank correlation coefficients between $\mathrm{NO}_{2}$ concentrations derived from each PDT preparation method and the continuous analyser are shown in Table 3 . The correlations within dipped PDT data (all $r>0.73$ ) and between dipped PDT data and analyser data ( $r$ values 0.58 $0.75)$ are consistently greater than the correlations within pipetted PDT data (all $r<0.67$ ) or between pipetted PDT data and analyser data ( $r$ values $0.55-0.68$ ). The poorer correlations associated with data from pipetting methods are probably partly a consequence of the lower precision associated with these data (Section 3.1), but must also reflect a greater inaccuracy of pipetting-method data to track the variation of $\mathrm{NO}_{2}$ concentration with exposure/location.

This latter observation is clearly evident in Fig. 2 which shows that, although PDTs prepared by all 8 methods show positive bias in $\mathrm{NO}_{2}$ concentration, there are systematic variations in the accuracy, i.e. the extent to which PDT measurements from different preparation methods are clustered together along a common trend. In general, $\mathrm{NO}_{2}$ data 

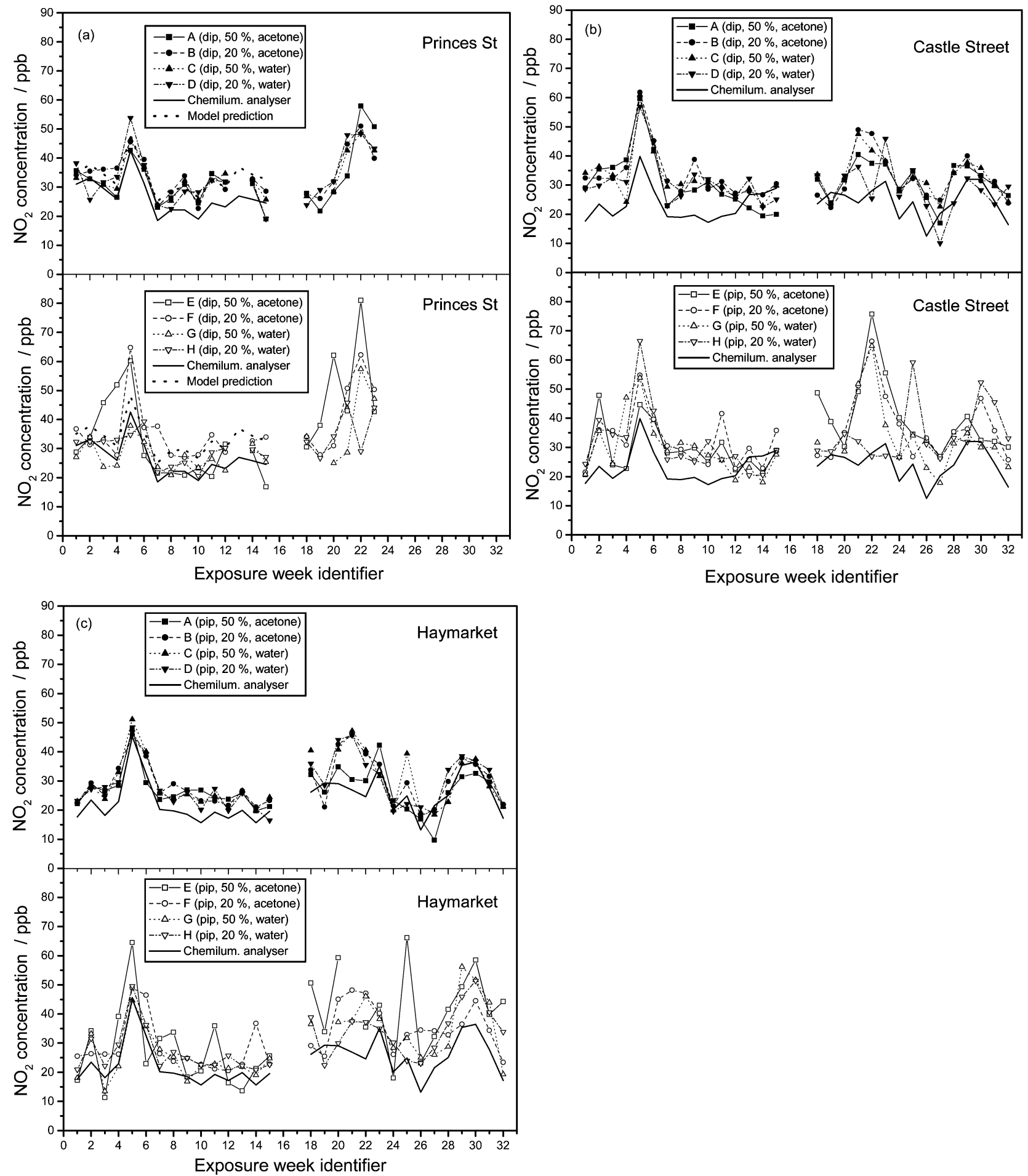

Fig. 2 Time-series of the mean (of duplicate) $\mathrm{NO}_{2}$ concentration determined from PDTs prepared by 8 different methods and exposed at 3 different urban locations in Edinburgh: (a) Princes St, (b) Castle St, (c) Haymarket. Also shown is the exposure-average $\mathrm{NO}_{2}$ concentration measured by co-located chemiluminescence analysers and, for the Princes St site, the $\mathrm{NO}_{2}$ concentration determined by a computer model of PDT sampling that also incorporates reaction within the tube between $\mathrm{NO}$ and $\mathrm{O}_{3}$. Identifiers 1-15 and 18-32 correspond to 1 week exposure periods between Nov 01-Mar 02, and Nov 02-Mar 03, respectively.

derived from PDTs prepared by dipping methods A-D (solid symbols) are both more closely clustered to each other, and follow a more tightly-constrained trend of values with respect to the analyser values, than the $\mathrm{NO}_{2}$ data from PDTs prepared by pipetting methods $\mathrm{E}-\mathrm{H}$ (open symbols). These latter data are considerably more erratic in accuracy. Fig. 2 shows that data from method $E$ (but also methods $F$ and $G$ ) are particularly erratic. However, there needs to be a lot of caution in making statements concerning the accuracy of method $\mathrm{E}$ in particular, since the very poor precision associated with this method (Fig. 1b) implies very low confidence in the absolute values.

The existence of differences in $\mathrm{NO}_{2}$ concentration with tube preparation method is confirmed statistically $(P<0.001)$ by the non-parametric Friedman test, in which the $\mathrm{NO}_{2}$ values from the 8 preparation methods from each exposure are ranked from 1-8 and the sum of the ranks assigned to each method over the 76 complete sets compared. The preparation method(s) that differ significantly are determined by comparing the differences between the rank sums for the methods with an 
Table 3 Spearman rank correlation coefficients between $\mathrm{NO}_{2}$ concentrations determined by each PDT preparation method and by co-located chemiluminescence analyser. Each correlation is based on $\geqslant 71$ data.

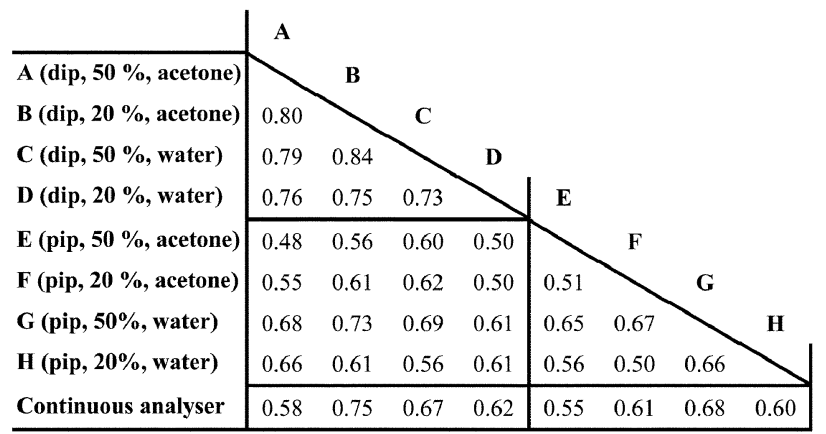

appropriate critical value (Table 4). The table shows that $\mathrm{NO}_{2}$ concentrations from preparation method $G$ are consistently lower than for other methods and significantly lower than for methods $\mathrm{F}$ and $\mathrm{B}$.

\section{Discussion}

The explanation of an intrinsic potential positive bias in PDT measurement of $\mathrm{NO}_{2}$ in locations at which $\mathrm{NO}, \mathrm{NO}_{2}$ and $\mathrm{O}_{3}$ are not at photostationary state is previously documented. ${ }^{5-7}$ This positive bias is again evident in the PDT data presented here (Fig. 2), and is again consistent with the chemical overestimation interpretation for the subset of data for which such a comparison can be made. It is important to remember that data here are from 1 week exposures. The fact that a positive bias often appears not to exist for PDTs deployed for longer exposures has been explained as the consequence of two opposing effects: a positive bias from chemical overestimation, and a negative bias from length of exposure. ${ }^{5,7}$ The latter was again demonstrated during this study through observation of significantly lower $\mathrm{NO}_{2}$ concentrations from 4 week exposed PDTs compared with the average of the four corresponding 1 week exposed PDTs. (Data not presented since they are not relevant to the main study on effect of PDT preparation method).

The comparison of PDT absorbent preparation methods shows a very clear tendency for data from PDTs prepared by dipping the grids in TEA-solvent prior to tube assembly to be more precise than data from PDTs prepared by pipetting the TEA-solvent on to the grids after tube assembly (Fig. 1). There is also a persistent tendency for data from PDTs prepared by pipetting to show greater fluctuation in accuracy relative to each other and to continuous analyser measurements (Fig. 2

Table 4 Pairwise differences in the Friedman rank sum of $\mathrm{NO}_{2}$ values obtained from PDTs prepared by methods A-H. (Differences expressed as rank sum for method in the row minus the rank sum for the method in the column). Exceedances of the Bonferoni-adjusted critical difference value of $94(P=0.05, N=76, k=8)$ are highlighted in bold italic.

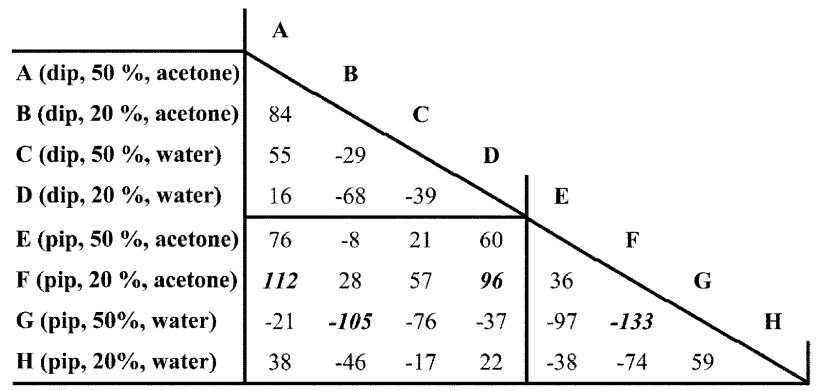

and Table 3). In so far as it is possible to make any statement regarding systematic trend in relative accuracy of preparation method (because of the demonstrable variation in precision with preparation method), it appears that method $\mathrm{G}$ (pipetting $50 \%$ TEA in water) generally yields the lowest concentrations, and method $\mathrm{F}$ (pipetting 20\% TEA in acetone) the highest (Table 4). Although the observation of a trend for lower $\mathrm{NO}_{2}$ concentrations derived from tubes prepared by pipetting $50 \%$ TEA in water on grids is consistent with the two previous investigations, ${ }^{8,9}$ it may also here be simply a statistical artefact arising from poorer precision.

All PDTs were subject to identical environmental conditions during each exposure, so factors such as chemical augmentation, shortening of diffusion path length, and exposuredependent degradation cannot explain differences in precision and accuracy associated with grid preparation method. Factors that can be postulated to contribute are the chemical conversion of $\mathrm{NO}_{2}$ at the absorbent, or the surface area over which $\mathrm{NO}_{2}$ absorption is assumed to occur.

The molar ratio of TEA added to the grids in any of the methods studied should be well in excess of the amount of $\mathrm{NO}_{2}$ collected during an exposure period. This was shown in this study by weighing the grids pre- and post-addition of the TEA solution, and is in agreement with similar calculations by Kirby et $a l .{ }^{8}$ Therefore, there should be sufficient TEA present for $100 \%$ conversion of $\mathrm{NO}_{2}$ whatever the method of preparation.

The mechanism of complexation in eqn. (1) was proposed by Glasius et al. ${ }^{11}$ to accord with the observation (using FAB-MS) that TEA N-oxide was the only TEA-derived product of the reaction. The mechanism can also be written stoichiometrically equivalently as:

$$
\begin{gathered}
2 \mathrm{NO}_{2}+\underset{ }{+} \mathrm{N}\left(\mathrm{CH}_{2} \mathrm{CH}_{2} \mathrm{OH}\right)_{3}+\mathrm{H}_{2} \mathrm{O} \rightarrow 2 \mathrm{NO}_{2}{ }^{-}+ \\
{ }_{\mathrm{O}-}{ }^{+} \mathrm{N}\left(\mathrm{CH}_{2} \mathrm{CH}_{2} \mathrm{OH}\right)_{3}+2 \mathrm{H}^{+}
\end{gathered}
$$

which makes the involvement of water more explicit. This version of the equation also does not imply the parallel formation of ${ }^{+} \mathrm{HN}\left(\mathrm{CH}_{2} \mathrm{CH}_{2} \mathrm{OH}\right)_{3}$ which might otherwise also be expected to be a TEA-derived product if TEA were required to yield formal $\mathrm{OH}^{-}$ions by base dissociation of water. The proposed mechanism is consistent with the known ability of tertiary amines to act as a reductant. The water molecules are assumed to derive from the air during exposure, or directly from water solvent if used in preparation of absorbent solution. However, the complexation method demonstrably works when acetone is the solvent. Since the acetone is presumed to evaporate rapidly from the grids when used as the TEA solvent, it appears there is sufficient flux of water molecules in the air to effect efficient $\mathrm{NO}_{2}$ complexation. Although some attempt has been made to explain the apparent lowering of TEA complexation efficiency for solutions prepared with $50 \%$ water as solvent ${ }^{8}$, it is not possible to rationalise how differences in complexation efficiency can arise for solutions of the same composition but applied by dipping or pipetting. Therefore, at this time, it is not feasible to explain how observed variations in PDT performance may be due to the chemistry of the $\mathrm{NO}_{2}$ complexation reaction alone.

The second factor to consider is surface area of absorption. When calculating the ambient $\mathrm{NO}_{2}$ concentration from the total $\mathrm{NO}_{2}^{-}$captured by a PDT it is assumed that $\mathrm{NO}_{2}$ is complexed by TEA absorbent across a surface area equal to the internal cross-section of the tube. It seems reasonable to assume that surface tension effects will ensure that a grid submerged in TEA-solvent solution will be consistently and evenly coated with solution on each occasion. In contrast, it seems less clear that using a pipette to introduce a small volume of solution onto grids within a cap (whether the tube has already been inserted into the cap or not) will always result in an absorbent surface area exactly equal to the value assumed in the calculation of $\mathrm{NO}_{2}$ concentration. Thus, failure to coat the 
entire grid surface with TEA will reduce the flux of captured $\mathrm{NO}_{2}$, leading to a negative bias in derived $\mathrm{NO}_{2}$ concentration, while any "creep" of solution up the inside wall of an assembled tube will increase the absorbent surface area and lead to a positive bias in derived $\mathrm{NO}_{2}$ concentration. The latter may occur in the pipetting methodology if tubes are tilted or inverted too quickly after pipetting and solution runs down the inside walls of the tube. (It is assumed that the physical act of patting dry dipped grids prior to assembly means that there is no possibility of the above issues for dipped preparation methods). Thus it is proposed that variation from one PDT to the next in the practical action of assembling tubes via a pipetting methodology may explain the greater imprecision of data derived from these preparation methods. The two pipetting methods that yield least precision (methods $\mathrm{E}$ and $\mathrm{G})$ both use $50 \%$ TEA solutions. Pure TEA is extremely viscous at room temperature, which is the reason it has to be dissolved in a solvent in the first place. A 50\% TEA solution is still fairly viscous, so the greater difficulty in reproducibly dispensing such a viscous solution from the pipette may also contribute to the greater imprecision for these specific preparation methods. In the work presented here, tubes were prepared by two different analysts, so whatever the specific cause(s) of the greater imprecision in pipetting methods, it was not analyst specific.

Finally, it is important to emphasise that any effect of preparation method on PDT precision and accuracy will be independent of the effect of any other operational factor(s) that may influence PDT accuracy (for example, a decline in measured $\mathrm{NO}_{2}$ from longer exposure, and/or an enhancement of measured $\mathrm{NO}_{2}$ from within-tube chemistry, or wind-induced turbulence). The $\mathrm{NO}_{2}$ measurement derived from a PDT exposure will be the composite of all relevant influences. The identified influences of these factors do not negate the continued use of PDTs for indicative $\mathrm{NO}_{2}$ measurement, but it is important that PDT data are always interpreted with due consideration of all parameters associated with their exposures.

\section{Conclusions}

$\mathrm{NO}_{2}$ concentrations derived from PDTs prepared by pipetting TEA-solvent solutions onto the grids after assembly are, in general, less precise than concentrations from PDTs prepared by dipping the grids into TEA-solvent solutions prior to tube assembly.

$\mathrm{NO}_{2}$ concentrations derived from PDTs prepared by pipetting TEA-solvent solutions onto the grids after assembly are also, in general, more variable in accuracy than concentrations from PDTs prepared by dipped methods. There is only weak evidence that one or more method gives systematically different $\mathrm{NO}_{2}$ across all exposures.
For 1 week exposures at these roadside and urban centre locations, $\mathrm{NO}_{2}$ concentrations from PDTs prepared by all methods are greater than chemiluminescence analyser $\mathrm{NO}_{2}$ concentrations, with greater discrepancy at the roadside locations.

Overall, it is concluded from this study that PDT performance is influenced by the physical method of grid preparation, whereby pipetting absorbent solution onto grids after tube assembly (particularly pipetting solutions of high $(50 \%)$ TEA composition) leads to an apparent degradation in precision and accuracy of $\mathrm{NO}_{2}$ measurement. It is recommended that these preparation methods be avoided, or at least investigated further.

\section{Acknowledgements}

We are grateful to Laura Macdonald (University of Edinburgh), for help in preparation and analysis of some of the diffusion tubes, and to Janet Brown (City of Edinburgh Council), for access to sites and provision of chemiluminescence analyser data.

\section{References}

1 E. D. Palmes, A. F. Gunnison, J. DiMattio and C. Tomczyk, Am. Ind. Hyg. Assoc. J., 1976, 37, 570.

2 K. Stevenson, T. Bush and D. Mooney, Atmos. Environ., 2001, 35, 281.

3 T. Bush, S. Smith, K. Stevenson and S. Moorcroft, Atmos. Environ., 2001, 35, 289.

4 A. J. Gair, S. A. Penkett and P. Oyola, Atmos. Environ., 1991, 25, 1927.

5 M. R. Heal, M. A. O’Donoghue and J. N. Cape, Atmos. Environ., 1999, 33, 513.

6 C. Kirby, M. Fox, J. Waterhouse and T. Drye, J. Environ. Monit., 2001, 3, 150.

7 M. R. Heal, C. Kirby and J. N. Cape, Environ. Monitor. Assess., 2000, 62, 39.

8 C. Kirby, M. Fox and J. Waterhouse, J. Environ. Monit., 2000, 2, 307.

9 A. Loader, Investigation of the effects of preparation technique on performance of nitrogen dioxide diffusion tubes, NETCEN report AEAT/ENV/R/0563, Harwell, UK, 2001

10 D. H. P. Laxen and P. Wilson, Compilation of diffusion tube collocation studies carried out by local authorities, UK Air Quality Archive, http://www.airquality.co.uk/archive/reports/cat06/ NO2DiffusionTubePerformance(Final).pdf, 2002.

11 M. Glasius, M. F. Carlsen, T. S. Hansen and C. Lohse, Atmos. Environ., 1999, 33, 1177.

12 E. D. Palmes and E. R. Johnson, Am. Ind. Hyg. Assoc. J., 1987, 48, 73.

13 M. R. Heal and J. N. Cape, Atmos. Environ., 1997, 31, 1911.

14 D. H. P. Laxen and E. Noordally, Atmos. Environ., 1987, 21, 1899.

15 D. Shooter, P. Brimblecombe, J. Shooter, D. Lowe, P. J. Day and S. Du, Environ. Technol., 1997, 18, 243. 